\title{
Subsidence and Upper-Tropospheric Drying along Trajectories in a General Circulation Model
}

\author{
Eric P. Salathé JR. and Dennis L. Hartmann \\ Department of Atmospheric Sciences, University of Washington, Seattle, Washington
}

(Manuscript received 2 October 1998, in final form 14 January 1999)

\begin{abstract}
A trajectory analysis of the Community Climate Model version 3 (CCM3) moisture simulation is used to show that the model simulates upper-tropospheric moisture observations better than would be inferred from a traditional geographical comparison. The upper-tropospheric moisture simulation is compared to upper-tropospheric moisture derived from Geostationary Operational Environmental Satellite 6.7- $\mu \mathrm{m}$ observations for September 1992. Trajectories start in convective regions of the Tropics and are followed into nonconvective subsidence regions. Moisture and pressure along the trajectories are determined for both the model and observations. Humidity values as a function of subsidence agree much better between observations and model than do geographical grid box comparisons, because the model does not simulate details in the large-scale flow pattern precisely. The relative humidity decreases slightly more slowly with subsidence along trajectories in the CCM3 simulation than in observations.
\end{abstract}

\section{Introduction}

The natural variability of upper-tropospheric humidity and the ability of atmospheric models to simulate it correctly both remain uncertain, and these uncertainties limit the confidence in climate simulations by atmospheric models. In this paper, we present a trajectory method for comparing simulated and observed uppertropospheric moisture. This method provides a different test for evaluating the ability of models to simulate the role of water vapor in climate change from comparisons made previously (Salathé et al. 1995; Soden and Bretherton 1994; Roca et al. 1997; Schmetz and Van de Berg 1994). The trajectory method reveals the physical relationship between upper-tropospheric humidity and the large-scale subsiding flow, which appears to be important in determining the climate response to perturbations in convective intensity and frequency. Thus, accurately modeling the distribution of tropical convection and the relationship between convective moistening and subsidence drying along trajectories is critical to satisfactorily simulating the tropical climate.

Salathé and Hartmann (1997) present a trajectory analysis of upper-tropospheric humidity (UTH) and show that the variation of UTH along trajectories provides a simple explanation of the horizontal moisture

Corresponding author address: Dr. Eric P. Salathé Jr., Department of Atmospheric Sciences, University of Washington, Seattle, WA 98195-1640.

E-mail: salathe@atmos.washington.edu distribution away from convection. Similar studies by Pierrehumbert (1998) and Soden (1998) have supported these results. The trajectory analysis separates the effect of large-scale advection on clear-air moisture from details in the large-scale wind pattern and in the location and intensity of convection. In this paper we will use this trajectory analysis to compare observations with simulations by the National Center for Atmospheric research (NCAR) Community Climate Model version 3 (CCM3). The simulation compares much better with observations when the trajectory analysis is used than when traditional Eulerian averages are used.

The comparison of atmospheric simulations with observations poses many challenges as discussed by Randall and Wielicki (1997). Geographical pattern comparisons have limited scope in assessing the simulation of atmospheric processes in a general circulation model (GCM). A model can do well in producing the longterm mean moisture pattern yet fail to capture the physically important features that are reflected only at shorter timescales. For example, Salathé et al. (1995) showed that the Goddard Laboratory for Atmospheres (GLA) GCM satisfactorily captured the extreme contrast in moisture across the Tropics revealed in 10-yr means. The model, however, was unable to adequately simulate the moisture patterns observed for the 1983 El Niño SST anomaly. Thus, comparisons of long-term mean statistics may pose too weak a test of a climate model's ability to simulate climate perturbations. The reverse may also be true when comparisons of geographical patterns are too stringent a test. A model that only 
coarsely represents present-day statistics, but which accurately simulates the processes that determine the climate, might be quite useful for climate prediction. Longterm means may not reflect how well important processes are captured when there is an unrelated deficiency that, for example, mislocates convective activity.

A method to examine the global response of an atmospheric simulation is presented in a study by Sun and Held (1996), who compare the correlation between temperature and humidity in radiosonde data and a GCM simulation. They found that the simulation gave a much stronger coupling between moisture and temperature than observed, which they attribute to excess vertical moisture transport in the nine-level model.

In another study that separates physical relationships from their geographical locations, Fu et al. (1997) compared the moisture response of CCM2 simulated for the 1987 El Niño. By examining the correlation of UTH at all points in the Tropics with precipitation over the equatorial Pacific, they revealed the global moisture response to the shift in convection during El Niño. Their results show that the model can simulate the observed shift of extreme dry zones that surround tropical precipitation, in contrast to the GLA GCM results mentioned above.

The recent studies of the variation of upper-tropospheric moisture along trajectories (Salathé and Hartmann 1997; Pierrehumbert 1998; Soden 1998) appear to capture important processes controlling upper-tropospheric water vapor, and separate these processes from details of the circulation pattern. If the model can capture behavior comparable to these observational results, we can conclude that the model adequately represents many of the important physical mechanisms controlling upper-tropospheric water vapor and its relationship to convection. The ability of the model to accurately simulate climate processes and feedbacks is much more important to simulating climate change than is capturing details of the weather patterns.

\section{Data}

The observed data in this study is the same dataset presented in Salathé and Hartmann (1997). UTH, defined as the mean relative humidity with respect to water between 500 and $200 \mathrm{mb}$, is retrieved from Geostationary Operational Environmental Satellite (GOES) 6.7$\mu \mathrm{m}$ observations following the method of Udelhofen and Hartmann (1995) and Schmetz and Turpeinen (1988). Cloud-free pixel values are averaged on a $2.5^{\circ}$ grid to yield the clear-sky equivalent UTH. The retrieval entails forward calculations using European Centre for Medium-Range Weather Forecasts (ECMWF) temperature and moisture profiles to create a lookup table mapping UTH to satellite brightness temperature.

The high-cloud fractional coverage indicates the location of deep convection and is computed by comparing the cloud-top temperature from GOES $11-\mu \mathrm{m}$ images with the ECMWF temperature field to find the fraction of a grid box where the cloud top is colder than the $300-\mathrm{mb}$ temperature. This threshold is equivalent to the choice of $235-\mathrm{K}$ cloud-top temperature used, for example, by Chen et al. (1996), and includes most of the highly reflective, optically thick clouds associated with active convection.

To perform trajectory calculations, we use horizontal winds from the ECMWF analyses. The trajectories follow the two-dimensional movement of columns within the 500-200-mb layer, and are computed using the mean winds over that layer. To track the subsidence of the air column as it follows this horizontal path, we compute vertical motion as the radiatively driven descent (Salathe and Hartmann 1997). Radiative fluxes are computed using the CCM2 column radiation model. Temperature and moisture profiles for this computation are taken from the ECMWF analyses, with the satellite-derived UTH substituted between 500 and $200 \mathrm{mb}$.

The simulation is a 15-yr integration of the NCAR CCM3 forced by observed monthly mean SST, a socalled Atmospheric Model Intercomparison Project (AMIP) run. The NCAR CCM3 is a spectral GCM with T42 (approximately $2.8^{\circ}$ lat $\times 2.8^{\circ}$ long resolution), 18 vertical levels, and 20-min time resolution, using semiLagrangian transport for moisture (Kiehl et al. 1996, 1998). The moist convection parameterization includes the deep cumulus scheme of Zhang and McFarlane (1995). Important changes from CCM2 include modifications to the simulation of radiative transfer, the boundary layer, and moist convection. We use the simulated winds and moisture for the trajectory analysis, and use the convective precipitation field to indicate the convectively active regions in the simulation. As with the observed data, vertical motion is taken as the radiatively driven diabatic descent, with the radiative cooling computed from the simulated temperature and moisture profiles.

To compare the simulation to the satellite-derived data, we compute the GOES water vapor channel radiance from the CCM3 temperature and moisture fields using the same forward model as is used in the retrieval method. Applying the retrieval scheme to the radiance computed from the model data returns the simulated UTH for comparison to the observations. The most appropriate method for evaluating atmospheric models is to simulate the satellite observations from the model results (Salathe et al. 1995; Soden and Bretherton 1994).

\section{Simulation of convective regions}

The trajectory analysis depends on selecting the trajectory origins in deep convection. However, we are using different parameters to indicate convection in the observations and simulation, and thus we will begin this section by defining how convectively active regions are chosen in the observations and simulation. We will then use these definitions to compare the observed and simulated distribution of convective activity. In evaluating 


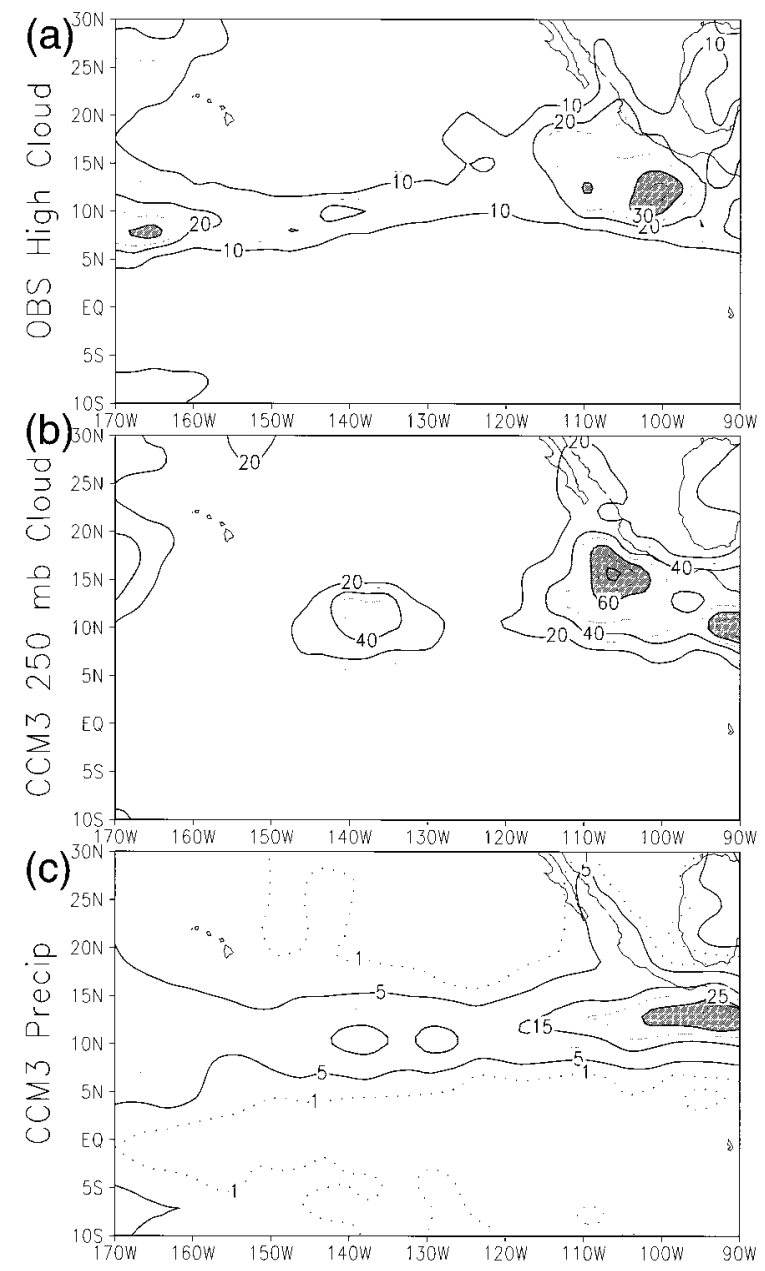

FIG. 1. The Sep 1992 monthly mean distribution of convective activity over the eastern tropical Pacific, (a) as indicated by observed high cloud fraction, (b) CCM3-simulated 250-mb cloud fraction, and (c) CCM3-simulated convective precipitation in $\mathrm{mm} \mathrm{day}^{-1}$.

the moisture simulation, we must consider two issues. The first, addressed in this section, is whether the model can produce the observed frequency and distribution of deep convection. The second, treated in sections 4 and 5 , is whether the model captures the correct relationship between convection and moisture transport into the clear air.

\section{a. Definition of convective regions}

Comparison between the observed and simulated cloud fraction presents several problems since this quantity is not defined in the same way in both cases. For the observations, we have defined the high cloud fraction as the proportion of satellite pixels in a $2.5^{\circ}$ grid box that indicate a cloud-top temperature colder than the ECMWF 300-mb temperature at that point. The monthly mean cloud fraction is shown in Fig. 1a. A partially cloudy pixel could be missed in this test if it results in a pixel mean less than the $300-\mathrm{mb}$ temperature.
Thus, the satellite-derived cloud fraction could underestimate the true high cloud fraction.

The monthly mean cloud fraction at each level is archived from the simulation. CCM3 diagnoses the cloud fraction at each pressure level based on the simulated vertical motion, humidity, and temperature (Kiehl et al. 1996). Ideally, to compare with the satellite-derived cloudiness, the overlapping clouds at each layer of the CCM3 simulation must be accounted for by some method. The maximum cloud fraction in the simulation occurs at $250 \mathrm{mb}$, so the cloud fraction at this level should be a reasonable indicator of the monthly mean high-cloud distribution, given the properties of deep convective cloud systems. The $250-\mathrm{mb}$ simulated monthly mean cloud fraction is shown in Fig. $1 \mathrm{~b}$.

Since only the monthly average and not the daily simulated cloud field is archived, we will use simulated convective precipitation to indicate locations of convection in the daily simulations. For the observations, we assume all points with more than $20 \%$ high cloudiness are convectively active. To find a threshold for the simulated precipitation that is consistent with high cloudiness, we compare the monthly mean simulated 250-mb cloudiness (Fig. 1b) and convective precipitation (Fig. 1c). The contour of $5 \mathrm{~mm} \mathrm{day}^{-1}$ precipitation covers a similar area of the eastern Pacific region as is covered by $20 \%$ simulated cloud fraction. Thus, we consider all points with convective precipitation greater than $5 \mathrm{~mm} \mathrm{day}^{-1}$ to be convectively active. Note that Fig. 1c indicates precipitation extending over the equatorial west Pacific; although the contours in Fig. 1b end at $10 \%$ cloudiness, there was cloudiness simulated with the precipitation there.

\section{b. Comparison of observations and simulation}

In the atmosphere, the location of convective activity is tightly coupled to SST gradients (Hartmann and Michelson 1993). Since the CCM3 simulation is forced by observed monthly mean observed SST, we expect that oceanic convection is simulated for the same locations as observed in the monthly mean. Figure 1a shows the mean observed high cloud fraction and Fig. 1b shows the monthly mean simulated cloud fraction at $250 \mathrm{mb}$ for September 1992. The monthly mean convective activity in the eastern Pacific is well located in the simulation. Cloud fraction, however, is considerably higher and less widespread than indicated by the observed cloudiness, even considering the discrepancies in how the two quantities are defined. Excess monthly mean precipitation in the CCM3 simulation for the East Pacific is consistent with excess cloud fraction (Hack et al. 1998).

With the thresholds for active convection chosen above (observed cloudiness exceeding 20\% and simulated precipitation exceeding $5 \mathrm{~mm} \mathrm{day}^{-1}$ ) we can now compare day-to-day variability of the simulated and observed convection. Since we cannot expect the model 
to duplicate the synoptic variability of convection, we shall compare observed and simulated values of the total area of the southeastern tropical Pacific occupied by convection, without reference to where within this region convection is located. Pierrehumbert (1995) has argued for the dynamical importance of the area occupied by convection in the Tropics. The convective area we might infer from the monthly mean cloud distribution will be different from the monthly average of the area on each simulated day, whereas the second is more physically relevant. We restrict the area of computation to the region from $30^{\circ} \mathrm{S}$ to $30^{\circ} \mathrm{N}$ and $170^{\circ} \mathrm{W}$ to $60^{\circ} \mathrm{W}$ and find the area of convective activity for each day in both the simulation and observations. Averaging the daily value of convective area over the month yields $26 \%$ for the simulation and $13 \%$ for the observations. Thus, the simulation based on the Zhang and McFarlane (1995) parameterization produces twice the instantaneous convective area than is typical for the observations. Also, the simulated deep convection tends to remain at fixed locations whereas in the observations the convective areas shift about the Tropics from day to day. Returning to the comparison of monthly mean cloudiness in Fig. 1, this behavior of the daily variability in convection is reflected by low values of the observed cloudiness spread over a large area compared to high values confined to the locations of the stationary convective activity in the simulation.

\section{Geographical moisture distribution}

Figure 2 displays the September 1992 mean distribution of upper-tropospheric humidity as derived from satellite observations (top), as simulated by CCM3 (middle), and the difference between the two (bottom). All grid points with active convection (i.e., cloud fraction over $20 \%$ for the observations and precipitation over $5 \mathrm{~mm} \mathrm{day}^{-1}$ for CCM3) were excluded from the monthly means to reduce the effects of cloud contamination. There may remain some cloud contamination of the observed UTH, which could yield a slight moist bias. The monthly mean observed and simulated wind vectors overlay the corresponding UTH distributions. The simulated UTH captures the overall pattern and extreme values that were observed, with moist values along $10^{\circ} \mathrm{N}$ associated with ITCZ convection and mean relative humidities well below $15 \%$ in the southern subtropics. The moist region in the simulation, however, is more zonally uniform than the observations and spreads less far to the south. The difference map reflects this disparity. Negative values near $5^{\circ} \mathrm{N}, 100^{\circ} \mathrm{W}$ indicate that the gradient from wet to dry is farther north in the simulation. Positive values near $10^{\circ} \mathrm{N}, 145^{\circ} \mathrm{W}$ result from variations in the observed moisture along the ITCZ. The discrepancy in the moisture patterns is very likely a result of a discrepancy in the observed and simulated upper-level winds. The observations show northeasterly winds over the South Pacific whereas the
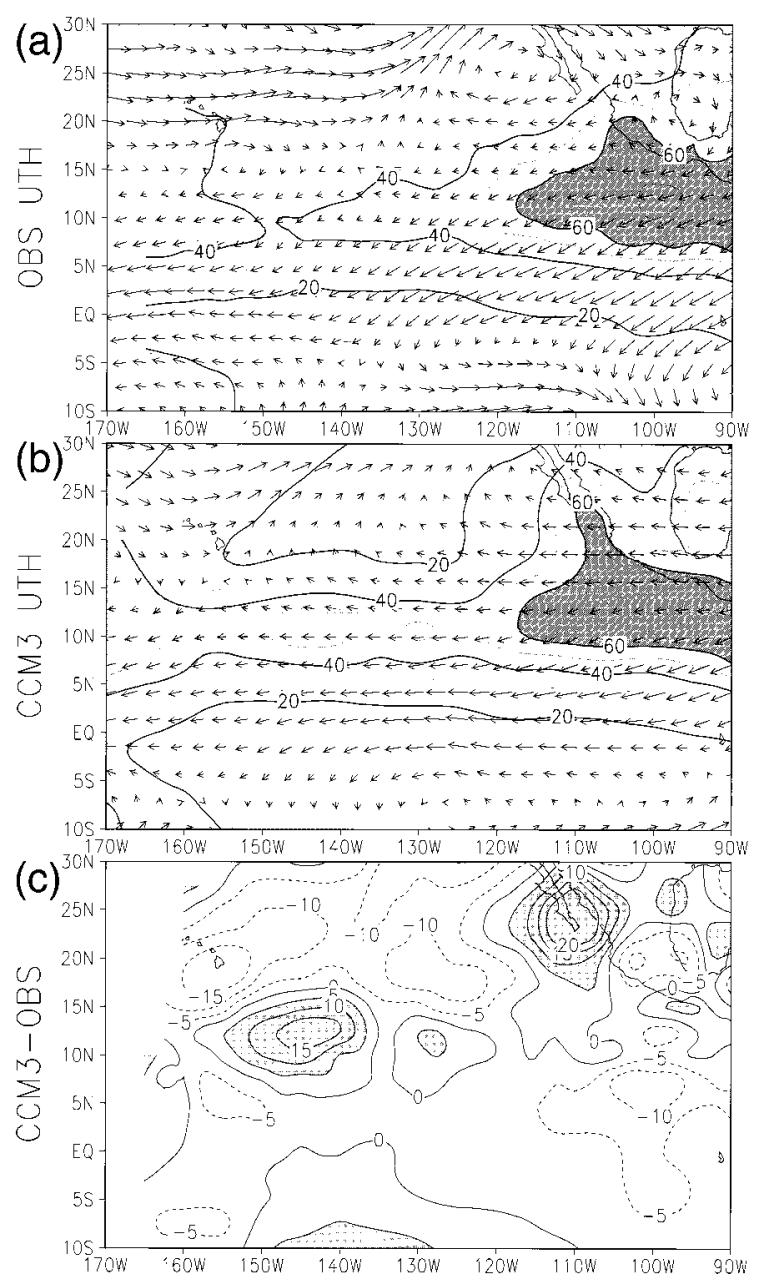

FIG. 2. (a) Observed and (b) CCM3-simulated UTH for the eastern tropical Pacific. Observed and simulated wind vectors overlay the humidity field. (c) Simulation minus the observation.

simulated easterlies are considerably more zonal. Note that the large differences at $5^{\circ} \mathrm{N}, 100^{\circ} \mathrm{W}$ correspond to an intensification of the northeasterlies in the observations. Hurrell et al. (1998) note a similar discrepancy in this region when comparing the $15-\mathrm{yr}$ mean winds simulated by CCM3 with observations for the southern winter (June-August).

Figure 3 (crosses) is a scatterplot of the observed and simulated monthly mean values at each grid point. Each cross is the average of the 30 daily simulated or observed UTH values in a given $2.5^{\circ} \times 2.5^{\circ}$ grid box. All convectively active grid boxes are excluded from the average using the cloud fraction and precipitation thresholds discussed above, and thus the scatterplot shows UTH values averaged only over convectively inactive grid boxes. A slight three-percentage-point dry bias in the simulated UTH is overwhelmed by scatter. The wide scatter reflects the discrepancies in the geographical distribution of moisture as discussed above. 


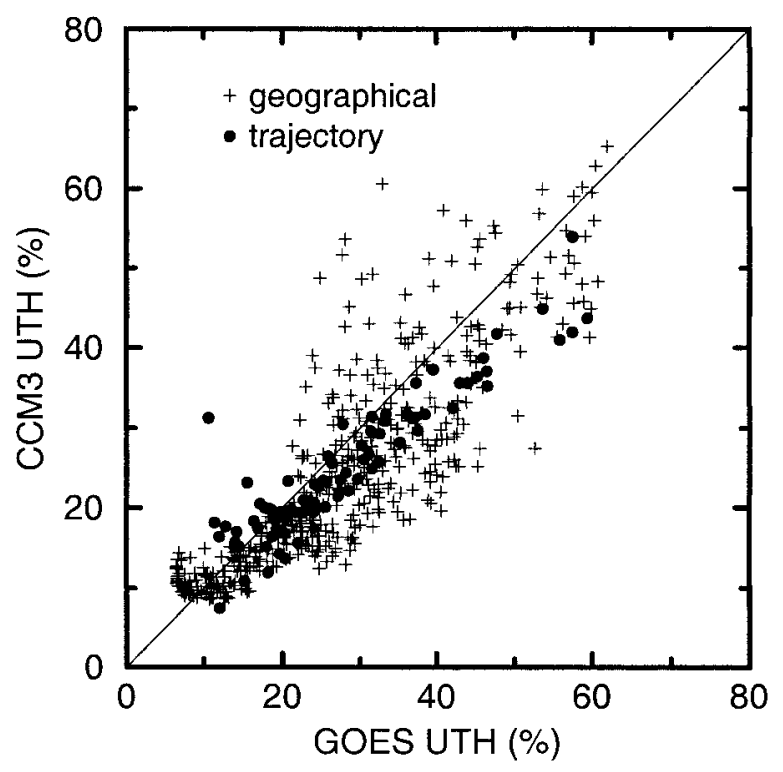

FIG. 3. Scatterplot of simulated and observed UTH values. Crosses indicate monthly mean of values at the same latitude and longitude. Circles indicate the mean of all values in the same interval of subsidence from convective source.

\section{Moisture variation along trajectories in observations and CCM3 simulation}

As discussed above, the recent observational studies by Salathé and Hartmann (1997), Pierrehumbert (1998), and Soden (1998) have shown that advection of moisture by subsiding motions gives a useful description of the observed tropical moisture distribution away from convection. We shall now explore whether CCM3 can duplicate these observed results. Success in duplicating this behavior will indicate that the model can capture processes that are essential to the large-scale relationship between tropical convection and upper-tropospheric moisture.

Following the method described in Salathé and Hartmann (1997), we compute trajectories of the 500-200$\mathrm{mb}$ layer that originate in deep convective systems during September 1992. Trajectories are computed forward from the convective source and the vertical velocity is found at each step from a radiative cooling calculation (see section 2). The vertical velocity is integrated along the trajectory to give the net vertical subsidence for all points along the trajectory. In general, multiple trajectories can pass through a single grid box if they converge. Thus, the net subsidence since exiting convection for air in a grid box can be found by averaging the subsidence values for all trajectories points that lie in the grid box. We then match the observed UTH to the net subsidence in each grid box at each time. The relationship between UTH and subsidence for the observed data (satellite-derived UTH and ECMWF winds) is shown by the crosses and dashed line in Fig. 4 and represents a composite view of subsidence and drying

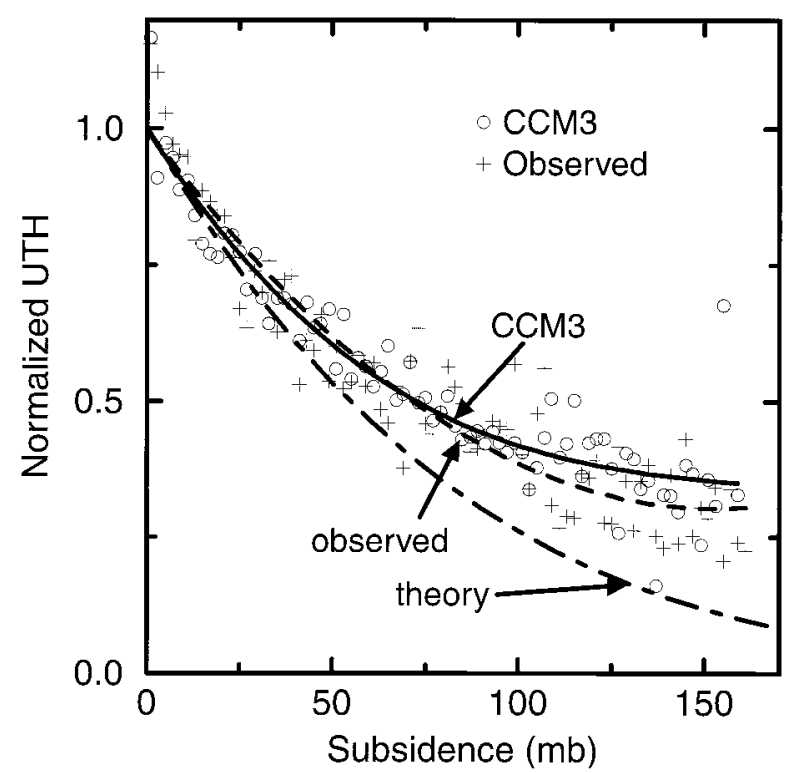

FIG. 4. UTH as a function of subsidence. Circles and crosses are means in 2-mb subsidence intervals for the simulation and observation; solid and dashed line are a cubic fit to the data. Dash-dot line is the theoretical relationship.

along a two-dimensional trajectory. Each cross represents the normalized average UTH in the 2-mb subsidence interval centered at that point. The dashed line is a cubic fit to the data to help visualize the relationship. The UTH values are normalized by a factor of 55\%, which is the average observed UTH in the convective region (i.e., for zero subsidence), so that the values represent the fractional decline in relative humidity since leaving convection

While the relationship between UTH and subsidence depends upon wind analyses that are influenced by the ECMWF atmospheric model, this relationship nevertheless represents our best estimate of the actual state of the atmosphere. The only ECMWF field that the results heavily depend upon is the upper-level horizontal winds. Moisture and convective activity come from satellite observations. Temperature only weakly influences the analysis. Vertical velocity, which is derived from the observed UTH and ECMWF temperature and moisture through radiative transfer calculations, varies little from typical tropical values. The ECMWF upper-level horizontal wind analyses are the field most tightly constrained by observations and most consistent with observations.

To test whether the GCM correctly simulates this relationship between subsidence and upper-tropospheric moisture, we repeat the analysis using the simulated winds and UTH for the same month. As with the observed data, all trajectory values falling into a grid box are averaged to give a subsidence value at each grid box sampled by the trajectory analysis. The relationship between the simulated UTH and subsidence is shown by the circles and solid line in Fig. 4; as above, each 
circle is the normalized average UTH in a 2-mb subsidence interval and the solid line is a cubic fit. The simulated humidity in the convective regions is $46 \%$, which is the normalizing factor. We compare normalized UTH values from the observations and $\mathrm{CCM} 3$ since the difference in humidities found in the convective regions is very sensitive to the choice of the threshold values for convection, whereas these choices do not strongly affect the relationship between UTH and subsidence. Thus, comparing normalized UTH emphasizes the shape of the subsidence drying curves in Fig. 4. Nevertheless, the actual bias between observed and CCM3 UTH is fairly small, as can be seen in Fig. 3, where dimensional values are used. The CCM3 UTH is on average three percentage points dryer than the observations for the trajectory comparison, as it is for the geographical comparison.

Following Salathé and Hartmann (1997), the expected drying of the upper-tropospheric layer after exiting convection and subsiding can be computed by assuming that all parcels in a column of air subside together, specific humidity is conserved in each parcel, and the temperature adjusts to the environmental value by radiative cooling balancing adiabatic warming. Thus, if the specific humidity profile in the convectively active region is $s(p)$ and the environmental temperature profile is $T(p)$, then when an air parcel has subsided from $p_{1}$ to $p_{2}$, its specific humidity will be $s\left(p_{1}\right)$ and its temperature will be $T\left(p_{2}\right)$. Relative humidity will decrease as the parcel subsides since its specific humidity remains fixed while its temperature increases to the environmental value at the lower level. The radiative cooling required for a parcel to subside a certain pressure increment depends on the environmental temperature profile.

For each increment of subsidence, we find the humidity profile of the subsided column as described above and integrate relative humidity over the 500-200$\mathrm{mb}$ layer to obtain UTH as a function of subsidence. Choosing an initial specific humidity profile in the convective region to give $75 \%$ relative humidity at all levels, taken with respect to ice below $273 \mathrm{~K}$, we compute UTH as shown by the theoretical curve (dash-dot) in Fig. 4. The curve has been normalized by UTH $=58 \%$, which is the value of UTH for no subsidence (i.e., in the convective region). The observations and simulation follow similar drying curves, but both dry less quickly than the theoretical curve. The less rapid drying of the observed and simulated data compared to the theory likely reflects a breakdown of the simple theory at larger distances along the trajectory. The accumulation of small amounts of moisture injected by shallow convection could cause slower drying. Also, since the dry conditions at large subsidence values represent a small fraction of the total atmosphere, any mixing would be with younger, less subsided, air parcels and would have a relative moistening effect.

To further illustrate the similarity between the simulated and observed subsidence drying, in Fig. 3 (cir- cles) the observed UTH values are plotted against the simulated UTH values from the same subsidence interval; in this figure, values are not normalized to show the small bias that remains. Since the trajectories are limited to the clear sky, these points reflect only convectively inactive values, as for the crosses from the geographical comparison. UTH values are averaged in 2 -mb subsidence intervals so that each interval contains approximately 30 independent UTH values, corresponding to the 30 values in the monthly averages used for the geographical comparison. Thus, the circles and crosses represent averages over approximately the same number of independent data values.

By matching UTH values with the same amount of subsidence, the circles compare the two fields according to similar dynamical history rather than by simply occupying the same latitude and longitude as for the pairs marked by crosses. Again, no clear bias between the simulated and observed UTH can be seen, but the scatter is considerably reduced, implying a much better ability of the model to capture the observed UTH variability. However, the reduced scatter in Fig. 3 and the two curves in Fig. 4 reveal that the simulation does not produce sufficient moisture near convection and UTH does not decrease quite as rapidly away from convection as observed. The increased scatter at low UTH is likely due to the problems with the trajectory method at large distances from convection as noted in Salathé and Hartmann (1997). The trajectory calculation itself becomes uncertain at large distances and moisture injection by shallow convection or by subgrid-scale mixing may also be important. The results, however, show that, to a large extent, the CCM3 captures the observed rapid drying as air subsides away from convection.

\section{Conclusions}

Since this study has been based on only a single month of data over a small region, we cannot conclusively characterize the ability of the CCM3 to simulate subtropical water vapor. The western subtropical Pacific, however, is an ideal region for examining the mechanisms controlling water vapor since convection is persistent along the ITCZ and an extremely large and dry region forms in the Southern Hemisphere during September.

Comparing the geographical pattern of monthly mean observed and simulated UTH suggests the model can capture the overall monthly mean pattern of moisture and convection, but substantial discrepancies in the patterns imply deficiencies in the simulated moisture transport. These moisture discrepancies appear to coincide with differences in the simulated and observed largescale winds, however, and therefore may not indicate an important deficiency in the model physics, since it is a free-running climatology run and not a numerical weather prediction. Thus, the ability of the model to simulate the processes controlling moisture is masked 
by its inability to precisely simulate the location of dynamical features on a monthly timescale, given only the weak forcing of observed SST.

The Lagrangian depiction of moisture advection and subsidence presented in Salathé and Hartmann (1997), Pierrehumbert (1998), and Soden (1998) suggests a means of evaluating the simulated moisture that removes essential processes from the detailed weather patterns. Comparison of the simulated relationship between subsidence and UTH with the observational results from Salathé and Hartmann (1997) shows that CCM3 captures the observed relationship quite well. Comparing observed and simulated UTH for grid points with similar subsidence histories shows much better correlation between observations and simulation than comparing Eulerian averages. It appears, therefore, that CCM3 can simulate important processes linking tropical convection and the transport of moisture to the subtropics. Consequently, improvements in the moisture simulation will depend on advances in the simulation of convective regions and in the convective parameterization.

Acknowledgments. This research was funded by NASA Grant NAG5-6101 to the University of Washington and by subcontract PNO 34402 under NASA Grant NAG-1-1849 to Harvard University.

\section{REFERENCES}

Chen, S. S., R. A. Houze, and B. E. Mapes, 1996: Multiscale variability of deep convection in relation to large-scale circulation in TOGA COARE. J. Atmos. Sci., 53, 1380-1409.

Fu, R., R. E. Dickinson, and B. Newkirk, 1997: Response of the upper tropospheric humidity and moisture transport to changes of tropical convection. A comparison between observations and a GCM over an ENSO cycle. Geophys. Res Lett., 24, 23712374.

Hack, J. J., J. T. Kiehl, and J. W. Hurrell, 1998: The hydrologic and thermodynamic characteristics of the NCAR CCM3. J. Climate, 11, 1179-1206.

Hartmann, D. L., and M. L. Michelson, 1993: Large-scale effects on the regulation of tropical sea surface temperature. J. Climate, $\mathbf{6}$, 2049-2062.
Hurrell, J. W., J. J. Hack, A. Boville, D. L. Williamson, and J. T. Kiehl, 1998: The dynamical simulation of the NCAR Community Climate Model version 3 (CCM3). J. Climate, 11, 1207-1236.

Kiehl, J. T., J. J. Hack, B. G. Bonan, B. A. Boville, B. P. Briegleb, D. L. Williamson, and P. J. Rasch, 1996: Description of the NCAR Community Climate Model (CCM3). NCAR Tech. Note, NCAR/TN420+STR, 152 pp. [Available from National Center for Atmospheric Research, P.O. Box 3000, Boulder, CO 80307.]

,,$---\longrightarrow$, D. L. Williamson, and P. J. Rasch, 1998: The National Center for Atmospheric Research Community Climate Model: CCM3. J. Climate, 11, 1131-1149.

Pierrehumbert, R. T., 1995: Thermostats, radiator fins, and the local runaway greenhouse. J. Atmos. Sci., 52, 1784-1806.

_ 1998: Lateral mixing as a source of subtropical water vapor. Geophys. Res. Lett., 25, 151-154.

Randall, D. A., and B. A. Wielicki, 1997: Measurements, models, and hypotheses in the atmospheric sciences. Bull. Amer. Meteor. Soc., 78, 399-406.

Roca, R., L. Picon, M. Debois, H. Le Treut, and J.-J. Morcrette, 1997: Direct comparison of Meteosat water vapor channel data and general circulation model results. Geophys. Res. Lett., 24, 147150.

Salathé, E. P., and D. L. Hartmann, 1997: A trajectory analysis of tropical upper-tropospheric moisture and convection. J. Climate, 10, 2533-2547.

— D. Chesters, and Y. C. Sud, 1995: Evaluation of the uppertropospheric moisture climatology in a general circulation model using TOVS radiance observations. J. Climate, 8, 2404-2424.

Schmetz, J., and O. M. Turpeinen, 1988: Estimation of the uppertropospheric relative humidity field from Meteosat water vapor image data. J. Appl. Meteor., 27, 889-899.

_ servations from Meteosat compared with short term forecast fields. Geophys. Res. Lett., 21, 573-576.

Soden, B. J., 1998: Tracking upper tropospheric water vapor radiances: A satellite perspective. J. Geophys. Res., 103, $17069-$ 17081.

— bution in general circulation models using satellite observations. J. Geophys. Res., 99, 1187-1210.

Sun, D.-Z., and I. M. Held, 1996: A comparison of modeled and observed relationships between interannual variations of water vapor and temperature. J. Climate, 9, 665-675

Udelhofen, P. M., and D. L. Hartmann, 1995: Influence of tropical cloud systems on the relative humidity in the upper troposphere. Geophys. Res., 100, 2162-2174.

Zhang, G. J., and N. A. McFarlane, 1995: Sensitivity of climate simulations to the parameterization of cumulus convection in the Canadian Climate Centre general circulation model. Atmos.Ocean, 33, 407-446. 